\title{
Magnetic iron oxide nanowires formed by reactive dewetting
}

Article

Accepted Version

Bennett, R. A., Etman, H. A., Hicks, H., Richards, L., Wu, C., Castell, M. R., Dhesi, S. S. and Maccherozzi, F. (2018) Magnetic iron oxide nanowires formed by reactive dewetting. Nano Letters, 18 (4). pp. 2365-2372. ISSN 1530-6992 doi: https://doi.org/10.1021/acs.nanolett.7b05310 Available at https://centaur.reading.ac.uk/76189/

It is advisable to refer to the publisher's version if you intend to cite from the work. See Guidance on citing.

To link to this article DOI: http://dx.doi.org/10.1021/acs.nanolett.7b05310

Publisher: ACS Publications

All outputs in CentAUR are protected by Intellectual Property Rights law, including copyright law. Copyright and IPR is retained by the creators or other copyright holders. Terms and conditions for use of this material are defined in the End User Agreement.

\section{www.reading.ac.uk/centaur}

\section{CentAUR}

Central Archive at the University of Reading 
Reading's research outputs online 


\section{Magnetic Iron Oxide Nanowires Formed by}

\section{Reactive Dewetting}

Roger A. Bennett ${ }^{*}$, Haitham A. Etman ${ }^{1}$, Hannah Hicks ${ }^{1}$, Leah Richards ${ }^{1}$, Chen Wu ${ }^{2}$, Martin R. Castell $^{2}$, Sarnjeet S. Dhesi $i^{3}$ and Francesco Maccherozzi ${ }^{3}$

${ }^{1}$ Department of Chemistry, University of Reading, Reading, Berkshire, RG6 6AD, UK;

${ }^{2}$ Department of Materials, University of Oxford, Parks Road, Oxford, OX1 3PH, UK; ${ }^{3}$ Diamond Light Source Ltd, Diamond House, Harwell Science and Innovation Campus, Didcot, Oxfordshire, OX11 0DE, UK

$\dagger$ Present address: School of Materials Science and Engineering, Zhejiang University, Hangzhou 310027, China

$\mathrm{Fe}_{3} \mathrm{O}_{4}, \mathrm{Re}(0001)$, spintronics, XMCD, STM, XPEEM, LEEM, LEED, oxide ultra-thin films

The growth and reactive dewetting of ultra-thin films of iron oxides supported on $\operatorname{Re}(0001)$ surfaces have been imaged in-situ in real time. Initial growth forms a non-magnetic stable $\mathrm{FeO}$ (wüstite like) layer in a commensurate network upon which high aspect ratio nanowires of several microns length but less than $40 \mathrm{~nm}$ width can be fabricated. The nanowires are closely aligned with the substrate crystallography and imaging by X-ray magnetic circular dichroism shows that each contain a single magnetic domain. The driving force for dewetting appears to be 
the minimization of strain energy of the $\mathrm{Fe}_{3} \mathrm{O}_{4}$ crystallites and follows the Tersoff and Tromp model in which strain is minimized at constant height by extending in one epitaxially matched direction. Such wires are promising in spintronic applications and we predict that the growth will also occur on other hexagonal substrates.

The continued interest in developing electronic devices, in which the electron spin is manipulated to create spin polarized electron currents, has generated much fundamental research into materials, their interfaces, grain boundaries and defects. Of particular importance are the half-metallic ferromagnetic materials in which the electronic density of states should be as near $100 \%$ spin polarized at the Fermi level as possible to enable spin polarized transport and the development of magnetoresistance and spin valve type devices. In this class of materials magnetite $\left(\mathrm{Fe}_{3} \mathrm{O}_{4}\right)$ has become a leading prospect due to high spin polarization ${ }^{1}$ and a Curie temperature of over $800 \mathrm{~K}^{2}$. It also displays a Metal-Insulator Verwey transition at $123 \mathrm{~K}$ in which structural distortions lead to charge localization and to the formation of linear Fe ion trimerons ${ }^{3,4}$. The self-organisation of the charge and orbital states couples the spin order and magnetism to the Verwey distortion ${ }^{3}$. Both magnetization and structural distortions found in the bulk materials are modified in nanostructures with the Verwey transition suppressed below 20nm and disappearing at $6 \mathrm{~nm}$ sized nanoparticles ${ }^{5}$.

Controlled synthesis of magnetite nanostructures on surfaces or electrodes is important in the measurement of fundamental material properties and the ongoing development of spintronics ${ }^{6,7}$. Pulsed laser deposition has been demonstrated to grow magnetite nanowires aligned along $<110>$ out of the plane of an $\mathrm{Al}_{2} \mathrm{O}_{3}(0001)$ substrate ${ }^{8}$ due to mismatched thermal expansion 
coefficients between substrate and growing film. Above $873 \mathrm{~K}$ the compressive strain is sufficient to drive out of plane growth of $100 \mathrm{~nm}$ diameter nanowires from low angle grain boundaries between epitaxially grown thick $\mathrm{Fe}_{3} \mathrm{O}_{4}(111)$ oriented films creating a dense nanowire forest. Pyramidal magnetite nanocrystals ${ }^{9}$ can be formed by dewetting (at $1373 \mathrm{~K}$ ) an $\mathrm{Fe}_{3} \mathrm{O}_{4}$ film grown (at $673 \mathrm{~K}$ ) on $\mathrm{SrTiO}_{3}(001)$ and re-depositing $\mathrm{Fe}_{3} \mathrm{O}_{4}$ at high temperature, despite the large lattice mismatch $(-7.5 \%)$. The substrate orientation stabilizes the growth of a (001) oriented film and the dewetting process leaves (001)-oriented islands that act as seeding centers for the formation of square pyramids with (111) side facets exposed. The nanopyramid structure was reported to reduce the coercive field in comparison to conventional magnetite films, an effect that was dependent upon pyramid size.

On metal substrates wetting layers are far more common due to the larger difference in surface free energies between oxides and metals. Indeed the wetting of metals by oxides is the origin of the strong metal support interaction in catalysis in which surface reactive metal sites are modified, usually blocked, by a thin layer of oxide from the support moving over the metal. First proposed by Tauster in $1978^{10}$, and imaged shortly thereafter by transmission electron microscopy ${ }^{11,12}$ in real catalytic materials, the mobility and responsiveness of transition metal oxide materials to their environment has proven to be a cornerstone concept in catalysis. The more recent development of advanced scanning probe microscopies have allowed imaging and identification of these layers on model crystalline systems on a per nanoparticle basis for a number of oxides including $\mathrm{Fe}_{3} \mathrm{O}_{4}, \mathrm{TiO}_{2}$ and $\mathrm{SrTiO}_{3}{ }^{13}$. Model studies of this effect have led to a plethora of investigations of ultra-thin oxides grown on well-defined single crystal metal substrates ${ }^{14}$ to further understand the structure and behavior of these important material systems. 
In this letter we report the formation of magnetic iron oxide nanowires by dewetting from an epitaxial wetting-layer overcoated with $\mathrm{Fe}$ in a low oxygen overpressure. The nanowires grow aligned with the crystallographic directions of the substrate forming high aspect ratio straight wires. A thin wetting layer remains on the surface and only the wires show ferro(ferri)magnetism in X-ray magnetic circular dichroism (XMCD) imaging by photoelectron emission microscopy (PEEM). Cross sectional scanning tunneling microscopy (STM) experiments across the wires show dominant step heights consistent with the magnetite structure.

Full experimental details are given in SI and here we give brief comments on the methodology employed to grow, image and analyze the nanostructures. Experiments were performed in two ultra-high vacuum (UHV) systems; one based on beamline I06 of the Diamond synchrotron for LEEM, PEEM, XMCD and LEED; the second at the University of Reading for STM, LEED and XPS. The clean $\operatorname{Re}(0001)$ crystal was prepared by cycles of annealing at $1300 \mathrm{~K}$ in $\mathrm{O}_{2}$ and flash annealing in UHV to $2300 \mathrm{~K}$ following an established method ${ }^{15}$. Iron was deposited in-situ by electron beam physical vapor deposition and oxides formed by post deposition annealing at various temperatures and pressures of $\mathrm{O}_{2}$. Formation of nanowires required at least two deposition steps, the first to form an oxide wetting layer and a second thicker Fe overlayer which oxidizes to form the nanowires. The growth conditions and pertinent imaging parameters such as tunneling current $\left(\mathrm{I}_{\mathrm{t}}\right)$ and sample bias $\left(\mathrm{V}_{\mathrm{b}}\right)$ are given in the text and figure captions.

The formation of the first oxide layer was made by evaporation of Fe metal onto the $\operatorname{Re}(0001)$ surface maintained at an elevated temperature $(510-773 \mathrm{~K})$, followed by oxidation at $1 \times 10^{-7}$ mbar $\mathrm{O}_{2}$. The evaporator flux and completion time for a monolayer (ML, where we define $1 \mathrm{ML}$ as the highest density of Fe that can be grown on $\operatorname{Re}(0001)$ before a second layer forms) could be determined by the use of STM ${ }^{16}$ (and corresponding XPS and LEED measurements) in the 
Reading UHV system or by either LEED or direct LEEM imaging in the Diamond UHV system (Movie 1 in SI). Figure 1 shows the evolution of the surface as the Fe monolayer grows on the Re substrate imaged in-situ by LEEM at $17.25 \mathrm{eV}$ beam energy. This energy was chosen to highlight the atomic step edges as dark lines, and the approximately triangular Fe islands as dark features against the grey terraces. The LEED pattern from a $1 \mathrm{ML}$ Fe film, Fig.1b, does not display any superstructure spots which indicates that Fe grows pseudomorphically in the first monolayer. The LEED pattern is six-fold symmetric due to the A-B-A hcp stacking sequence of the substrate ${ }^{15}$ presenting both $\mathrm{A}$ and $\mathrm{B}$ terminations at the surface. The second layer of $\mathrm{Fe}$ forms approximately triangular islands rotated $60^{\circ}$ with respect to each other on alternating terraces which is also due to these two terminations. The two monolayer thick islands clearly show up brighter in X-ray absorption (XAS) imaging modes, Fig. 1(c), (where the image contrast is formed from the difference between the intensity at the $\mathrm{Fe} \mathrm{L}_{3}$ absorption edge and off resonance (pre edge) as would be expected due to the increased X-ray absorption at the $\mathrm{Fe} \mathrm{L}_{3}$ edge. The XMCD analysis of such films (not shown) show no discernable contrast indicating that these ultra-thin heteroepitaxial layers are not ferromagnetic.

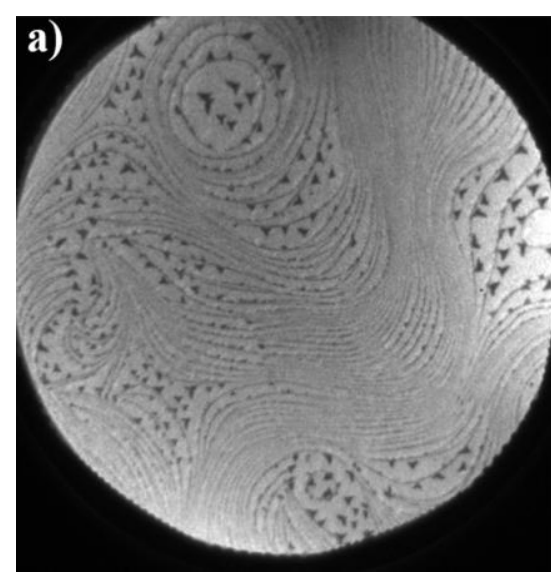

b)

Figure 1 . Fe deposition on $\operatorname{Re}(0001)$ at $500 \mathrm{~K}$ while LEEM imaging $(17.25 \mathrm{eV}, 15 \mu \mathrm{m}$ field of view (FOV)); a) surface structure at just over $1 \mathrm{ML}$ with crystallographic step edges showing as 
dark continuous lines and terraces as uniform grey and b) LEEM-LEED of this surface showing $(1 \times 1)$ pattern at $34.85 \mathrm{eV}$ beam energy. c) shows an XPEEM XAS intensity image with $15 \mu \mathrm{m}$ FOV taken as the difference between signal at $\mathrm{h} v=707.3 \mathrm{eV}$ on the $\mathrm{Fe} \mathrm{L}_{3}$ absorption edge and off resonance (pre edge) at $\mathrm{h} v=702 \mathrm{eV}$.

By heating such a Fe monolayer momentarily to $870 \mathrm{~K}$ while it is exposed to $1 \times 10^{-7} \mathrm{mbar}_{2}$ (and allowing it to cool in the gas) we generate a complete oxide wetting layer in registry with the substrate. A coincidence lattice forms composed of $8 \mathrm{FeO}$ overlayer (3.1 $\AA$ ) unit cells to $9 \mathrm{Re}$ substrate $(2.76 \AA)$ unit cells as shown in Figure 2. The structures seen in the STM are due to a Moiré pattern induced by a periodic fit of overlayer structures to the substrate over distances of approximately $2.48 \mathrm{~nm}$ with atomic scale features at shorter length scales. Grain boundaries and the change of terrace orientation at step edges give rise to alternative Moiré patterns and hence these features can image differently by STM (Fig. 2b). LEED patterns for 1ML oxidized films grown in the Reading STM laboratory (Fig. 2c) and in the Diamond I06 PEEM system (Fig. 2d) are in very good agreement and show the transferability of growth protocols between the two instruments. XPS indicates that the Re substrate is not oxidized, and we conclude that the LEED and STM results indicate that this FeO layer is structurally similar to that commonly found on other hexagonal metal substrates, for example on $\mathrm{Pt}(111)^{17,16}$ or $\mathrm{Ru}(0001)$, in which the $\mathrm{Fe}$ is on the metal with oxygen terminating to vacuum ${ }^{14 c, 18}$. XMCD measurements on these films (and those where a second layer of $\mathrm{FeO}$ had just begun to grow) show no magnetic structures. We note that $1 \mathrm{ML}$ wüstite films grown on $\mathrm{Ru}(0001)$, for which spin polarized LEEM measurements have been reported ${ }^{14 \mathrm{~d}}$, also do not show any magnetism. 


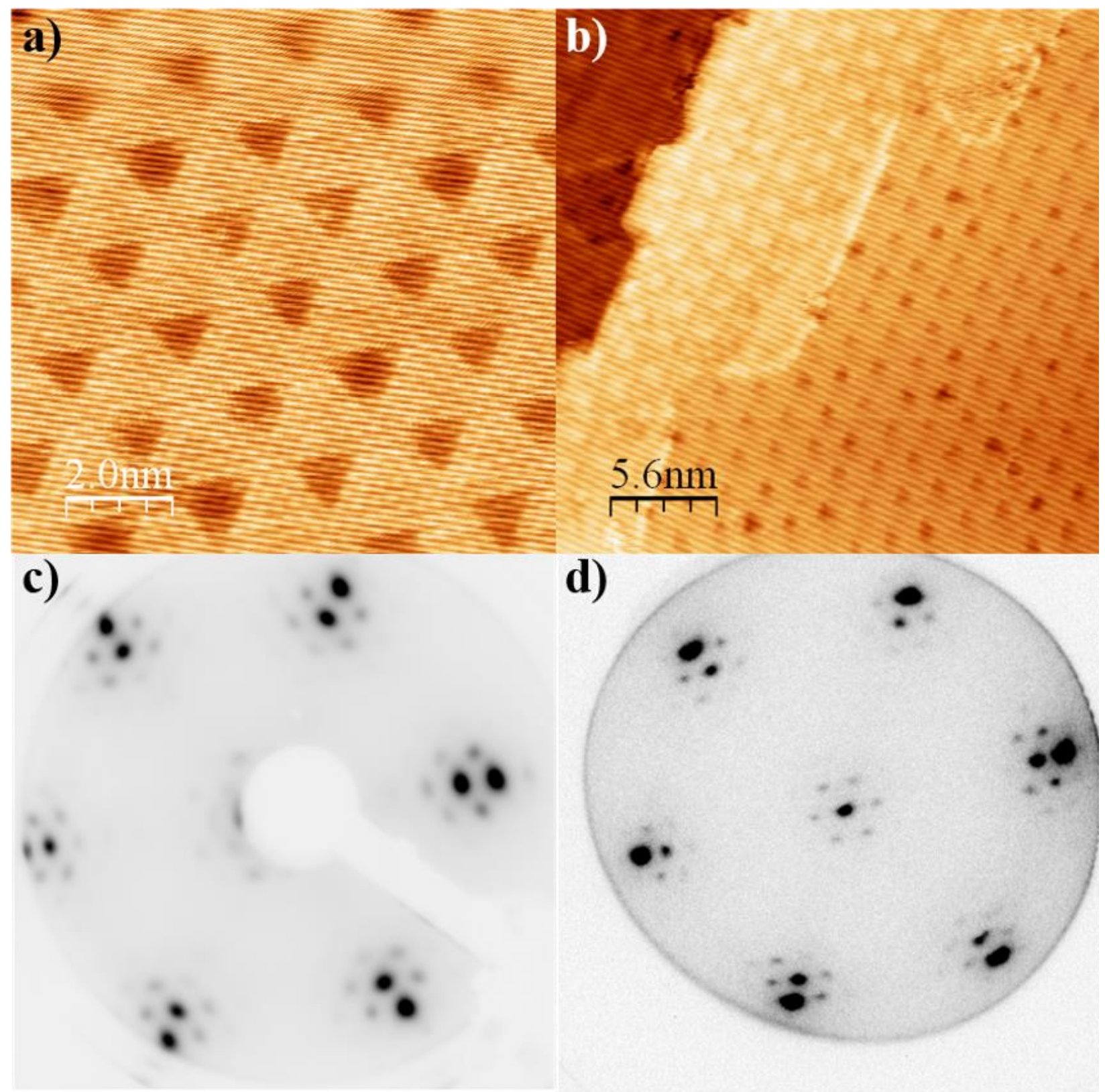

Figure 2. High resolution STM images of the $\mathrm{FeO}$ monolayer grown on $\operatorname{Re}(0001)$ showing periodic Moiré structure due to misfit of the overlayer to the substrate $\left(\mathrm{I}_{\mathrm{t}}=0.2 \mathrm{nA}\right.$ and $\mathrm{V}_{\mathrm{b}}=$ $0.1 \mathrm{~V})$. Step edges and grain boundaries between regions of the film change this misfit resulting in various appearances in $\operatorname{STM}\left(\mathrm{I}_{\mathrm{t}}=0.5 \mathrm{nA}\right.$ and $\left.\mathrm{V}_{\mathrm{b}}=1.5 \mathrm{~V}\right)$. c) Conventional LEED $(60 \mathrm{eV})$ and d) microspot LEEM/LEED (54eV) show near identical patterns from the FeO monolayer. 
To form nanowires we use this $\mathrm{FeO}(111)$ oriented monolayer as a substrate and deposit further Fe and oxidize again. Deposition in UHV of two or more monolayers of iron onto this wetting layer at temperatures between 300 and $373 \mathrm{~K}$ leads to the reduction of contrast in the LEED and the appearance of diffraction spots running from the $(0,0)$ spot as the beam energy is varied which is indicative of faceted structures (Movie 4 in SI). The LEEM images at this stage are also reduced in contrast but show no new features and we conclude that very small $3 \mathrm{D}$ iron rich islands are formed on the surface which are below the resolution of the LEEM. This result for the growth of $\mathrm{Fe}$ on $\mathrm{FeO}(111)$ films is similar to that for $\mathrm{Fe}$ on $\mathrm{Fe}_{3} \mathrm{O}_{4}(111)$ surfaces in which small nanoclusters initially nucleate followed by $3 \mathrm{D}$ growth ${ }^{19}$.

However, heating of such $\mathrm{Fe} / \mathrm{FeO} / \mathrm{Re}(0001)$ layered systems in an $\mathrm{O}_{2}$ pressure of $\sim 1 \times 10^{-7}$ mbar while slowly increasing the temperature leads to a remarkable change in surface morphology. Firstly, at $\sim 700 \mathrm{~K}$ a dynamic change to the whole surface occurs and is viewed in LEEM as a bright front progressing through the field of view. Such changes in LEEM imaging have recently been noted for mixed Fe oxide films on $\operatorname{Pt}(111)$ undergoing various phase transformations ${ }^{14 b, 20}$, such as compositional changes from $\mathrm{Fe}_{3} \mathrm{O}_{4}$ to $\alpha-\mathrm{Fe}_{2} \mathrm{O}_{3}$ (hematite) and for structural only transformations from $\gamma-\mathrm{Fe}_{2} \mathrm{O}_{3}$ (maghemite) to $\alpha-\mathrm{Fe}_{2} \mathrm{O}_{3}$. These studies were undertaken at higher oxygen pressures (to drive the formation of $\mathrm{Fe}_{2} \mathrm{O}_{3}$ ) than we use here. We find that the main structural events that occur to create nanowires happen after this reaction front has passed (Movie 2 in SI), and hence we will not comment further on this interesting phenomenon. Upon continued heating to $\sim 770 \mathrm{~K}$ the surface begins to rapidly restructure with terraces changing contrast dramatically in LEEM, and with the apparent dewetting and aggregation of islands that grow into the nanowires shown in Figure 3 (and as Movies 2 and 3 in SI). These nanowires are very long (typically up to several $\mu \mathrm{m}$ ) and narrow (a few tens of $\mathrm{nm}$ ) 
while crossing many step edges. They also appear to be closely aligned with the crystallography of the substrate and hence run in three dominant directions. On close inspection some wires are seen to have grown $10^{\circ}$ away from the principal growth direction and so two orientations are present $\pm 5^{\circ}$ from a substrate symmetry direction. It should be noted that without the additional $\mathrm{Fe}$, the $\mathrm{FeO}(111)$ on $\operatorname{Re}(0001)$ system on its own is stable up to these temperatures and slightly beyond so we conclude that additional Fe is crucial to the nanowire formation.

Figure $3 \mathrm{a}$ shows a $10 \mu \mathrm{m}$ field of view (FOV) LEEM image at $1.67 \mathrm{eV}$ of these nanowires in which they are seen to extend over several microns in length and across many step edges of the $\operatorname{Re}(0001)$ substrate without deviation. The remaining surface has a clearly defined step structure along with some regions between the wires in which the film has not fully dewet (appearing bright in the image). Figure $3 \mathrm{~b}$ shows the accompanying microspot LEED pattern at $100 \mathrm{eV}$ in which a very well defined $\mathrm{FeO}(111)$ on $\mathrm{Re}(0001)$ is the principal component (cf. Fig. 2c) along with some running spots arising from faceted structures on the surface. The small size of the nanowires and thermal drift of the sample stage precluded microspot LEED from a single nanowire and so these running spots may arise from inclined surfaces on both the nanowires and the incompletely dewet structures. Figure $3 \mathrm{c}$ shows a $6 \mu \mathrm{m}$ FOV XAS image in which the intensity is proportional to the total $\mathrm{x}$-ray absorption at the $\mathrm{Fe} \mathrm{L}_{3}$ edge (full sequence of frames in Movie 5 in SI). The high intensity nanowires are Fe rich in comparison to the intervening space between islands, however, some faint areas are visible between islands in which incomplete dewetting leaves residual clusters of small islands, in agreement with the LEEM in Fig 3a. The nanowires are therefore much thicker than the wetting layer and the residual islands.

The analysis of the XMCD image of the same area, Fig. 3d, suggests that all of the wires are monodomain and are magnetized along their principal axis. The value of the three distinct 
XMCD intensities that each nanowire may take (black, white or grey) arises from the dichroism effect being proportional to the cosine of the angle between the photon momentum and the local magnetization and is therefore compatible with the magnetization directions indicated by the redarrows in fig. 3d. Apparent junctions between nanowires visible in Fig. 3c are sharp and magnetically well defined in Fig. 3d. The non-magnetic surface between wires, characterised by zero XMCD signal, may be the result of a complete dewetting of Fe leaving bare non-magnetic Re surfaces or a remaining non-magnetic iron oxide wetting layer. The LEED pattern, however, shows that the $\mathrm{FeO}(111)$ structure dominates on the substrate surface indicating that a wetting layer of non-magnetic $\mathrm{FeO}(111)$ remains after dewetting of the excess $\mathrm{Fe}$ in the $\mathrm{O}_{2}$ environment. 

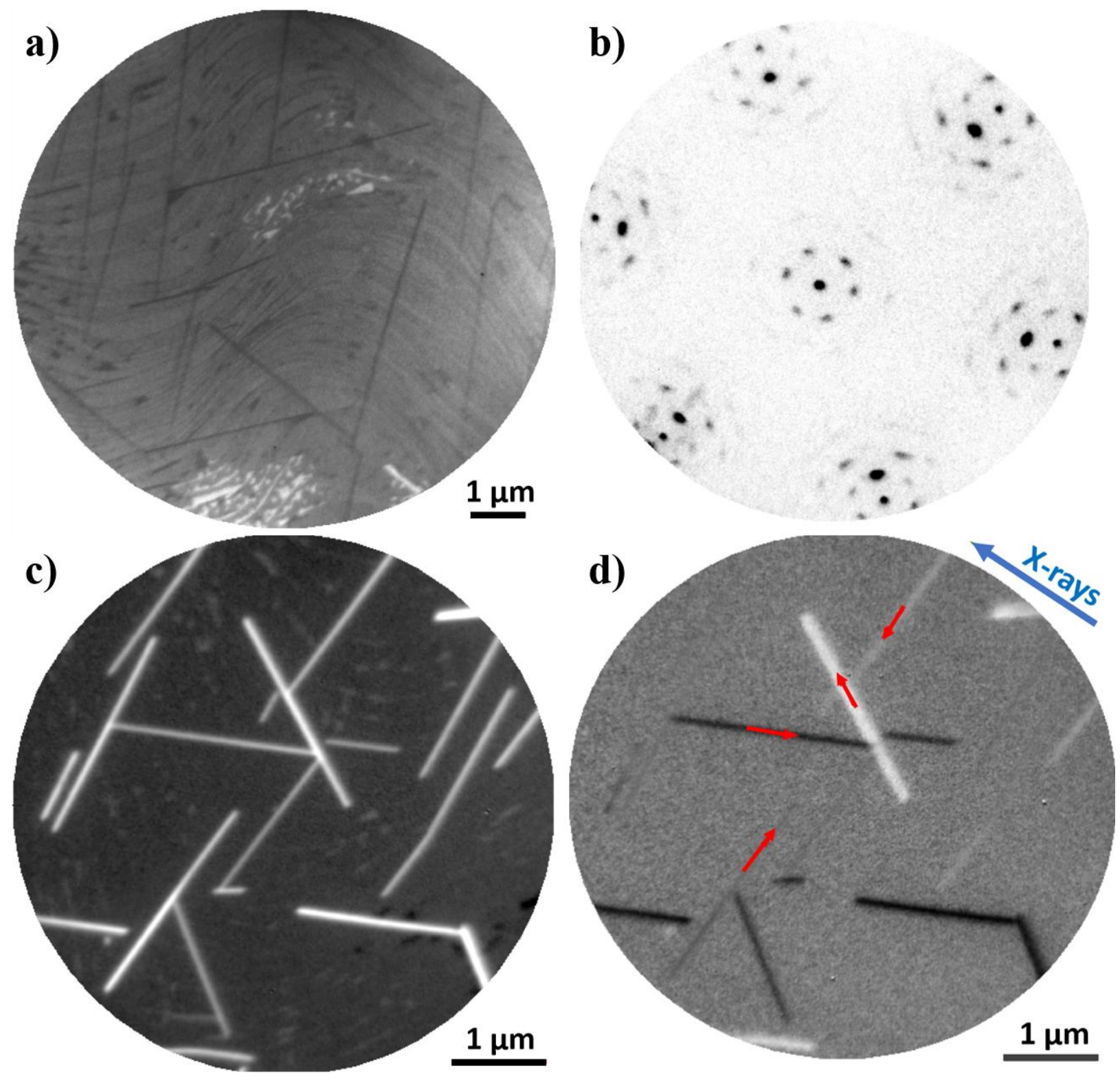

Figure 3. Real and reciprocal space images of the dewet nanostructures. Figure $3 \mathrm{a}$ is a $10 \mu \mathrm{m}$ FOV LEEM image at $1.67 \mathrm{eV}$ showing nanowires crossing substrate steps with smaller regions of bright and dark islands due to incomplete dewetting. Figure $3 b$ is a microspot LEED image taken at $100 \mathrm{eV}$ in which the wetting layer pattern is dominant with a superposition of very weak running spots and streaks (due to inclined planes of 3D structures on the surface). Figure $3 \mathrm{c}$ is a $6 \mu \mathrm{m}$ FOV image of the XAS signal and identifies the nanowires as Fe rich, and hence thick 
structures. Figure $3 \mathrm{~d}$ covers the same $6 \mu \mathrm{m}$ FOV as Fig. $3 \mathrm{c}$ but with contrast derived from the XMCD signal which indicates that each nanowire displays a single magnetic domain (see text for details, and SI for details on XMCD image formation methodology).

Having demonstrated that these nanowires are iron rich and single domain magnets we turn now to consider their composition and crystallography. Ferro (and ferri) magnetic iron containing structures could be pure iron which is ferromagnetic, $\mathrm{Fe}_{3} \mathrm{O}_{4}$ magnetite which is ferrimagnetic, or $\gamma-\mathrm{Fe}_{2} \mathrm{O}_{3}$ maghemite which is ferromagnetic, whereas other oxides of $\mathrm{Fe}$ will not display the observed magnetic signals. The oxygen pressure of $\sim 1 \times 10^{-7}$ mbar is insufficient to fully oxidise iron to $\mathrm{Fe}_{2} \mathrm{O}_{3}{ }^{21}$ but it is high enough to oxidise pure $\mathrm{Fe}$ to $\mathrm{FeO}$ or $\mathrm{Fe}_{3} \mathrm{O}_{4}$. We therefore expect magnetite to be the dominant magnetic oxide responsible for the nanowires. To test this hypothesis we employed XAS in the PEEM on our nanowire samples, and also on aluminium capped pure $\mathrm{Fe}$ and $\mathrm{Fe}_{3} \mathrm{O}_{4}$ single crystals. Doing this self-consistently on the same instrument allows direct comparison without having to calibrate photon energies from other sources as the literature contains a wide range of $\mathrm{Fe}_{2} \mathrm{~L}_{3}$ peak absorption energies for these materials ${ }^{22}$. For the nanowire samples we attempt to selectively extract data for the nanowires only and for the substrate uncovered between the dewet nanowires. However, the spatial resolution of the PEEM is comparable to the apparent width of the narrowest nanowires in the LEEM images ( $40 \mathrm{~nm})$ and so we expect some small contribution to the nanowire signal from the substrate but not vice versa. Our results are shown in Figure 4 and the XAS response for wires and wetting layer appear to be very similar (also see Movie 5 in SI). The XAS from all the nanowires in the field of view showed a maximal absorption at $707.4 \mathrm{eV}$, which is the same maxima found for the wetting layer on the substrate. For Fe metal films the Fe $\mathrm{L}_{3}$ peak was at $\sim 707 \mathrm{eV}$ with a long tail and the single crystal $\mathrm{Fe}_{3} \mathrm{O}_{4}$ peak was at $708.0 \mathrm{eV}$ without a long tail but 
with a small shoulder at $\sim 707 \mathrm{eV}$. From this we can see that the nanowire line shape and position differs from a metal considerably confirming that they have oxide character and therefore are not composed of pure Fe. The structure of the wires cannot be determined unequivocally from the XAS measurement.
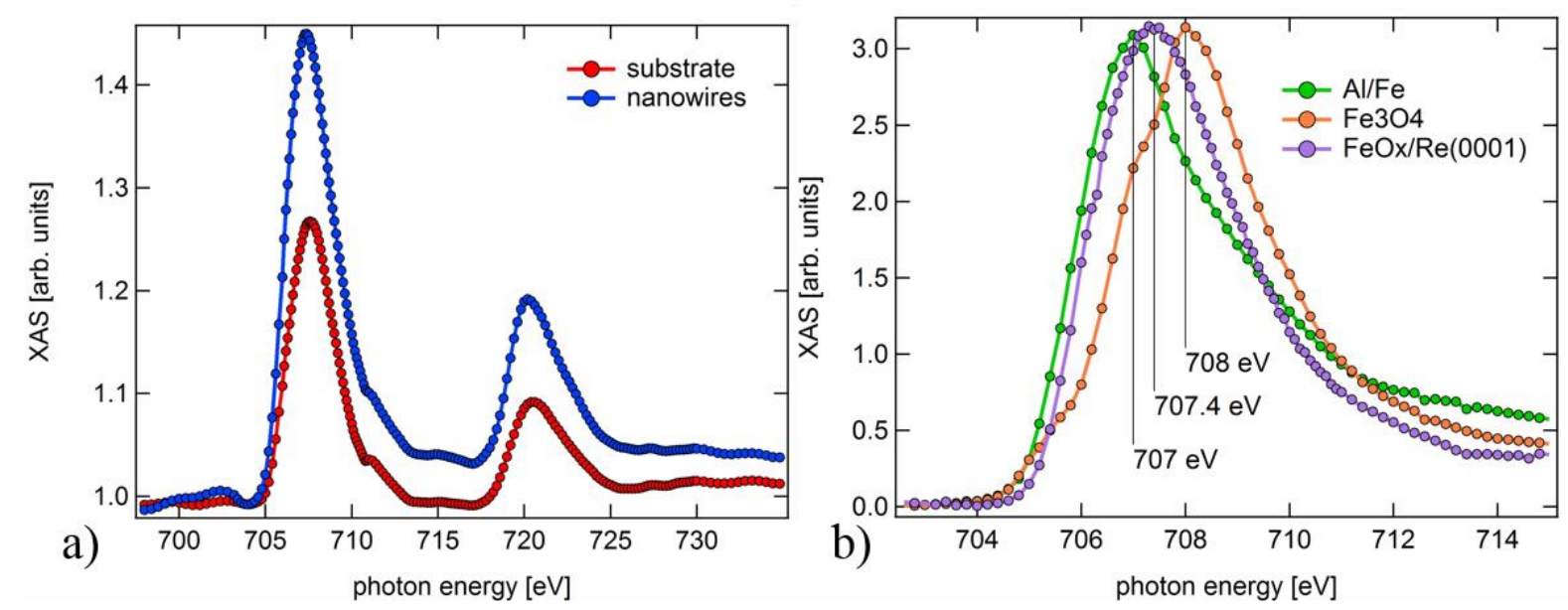

Figure 4. XAS at the Fe L absorption edges of a) nanowires only and the background substrate excluding nanowires for the whole image (with raw signal scaled to 1 pre-edge); b) a comparison between the nanowires and ( $\mathrm{Al}$ capped) pure $\mathrm{Fe}$ and a bulk $\mathrm{Fe}_{3} \mathrm{O}_{4}$ single crystal all measured on the same PEEM instrument on beamline I06.

The very small width and sparseness on the substrate is a problem for further identification by LEEM imaging and PEEM based spectroscopies and hence we return to STM imaging. The sparseness is still a problem as the imaging field of view is limited $(0.8 \mu \mathrm{m}$ maximum for our instrument - see SI fig. SI1 for a comparison of imaging scale to island density) but the potential advantage of the high spatial resolution of this microscopy is that it can provide atomic scale structural information. Unfortunately we also lose the significant ability of LEEM to image 
dynamically while heating and oxidising which allows intervention to freeze out the structures once they have been seen to have formed. Figure 5 shows the results of an STM experiment that follows the methodology used in the LEEM / PEEM experiments to form the nanowires. In Fig. 5a two nanowires can be seen running diagonally in the full field of view with other wires crossing opposite corners of the image out of the field of view. All of these structures show an extremely flat upper surface and run across the substrate steps that are just visible in the background (step heights of $0.23 \mathrm{~nm}$ corresponding to the Re interlayer spacing) without introducing steps in the wires upper surface. The structure to the top left is attached to a nanowire and appears to also contain multiple flat topped crystallites. We believe this is one of the residual islands apparent in LEEM in areas where dewetting to form nanowires is incomplete. The incomplete nature of the dewetting leads the nanowires to have stepped rather than fully close packed facetted sides. Close examination of two regions, highlighted by rectangular and square white boxes and shown in detail in Figs. 5b and 5c, identify that the steps all have a common height multiple of $0.48 \mathrm{~nm}$, which corresponds exactly to the interlayer unit cell repeat of magnetite $\mathrm{Fe}_{3} \mathrm{O}_{4}(111)$. The interlayer repeat of $\mathrm{FeO}(111)$ is $0.25 \mathrm{~nm}$ and for corundum structured $\alpha-\mathrm{Fe}_{2} \mathrm{O}_{3}(0001)$ it is $0.29 \mathrm{~nm}$. As these step heights, odd multiples of these steps or even multiples of $0.25 \mathrm{~nm}$ that would be close to multiples of $0.48 \mathrm{~nm}$ (but distinguishable) are not observed on the wires we conclude that the wires do not have either of these structures. After imaging this surface was removed from vacuum and imaged by scanning electron microscopy which confirmed the presence of wires with lengths and widths matching those seen at the maximum field of view of the STM, see SI fig. SI1. 

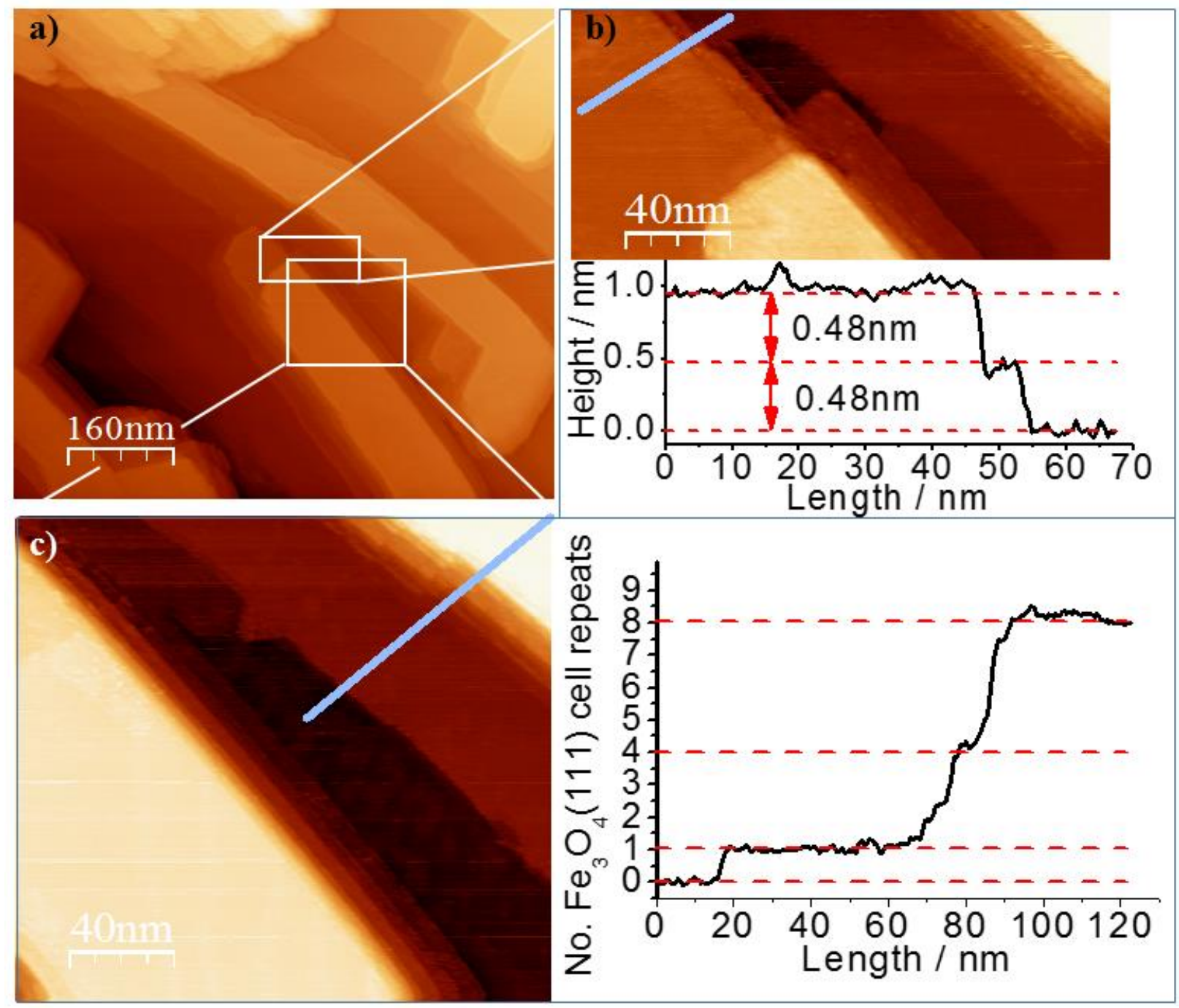

Figure 5. STM images $\left(\mathrm{I}_{\mathrm{t}}=1 \mathrm{nA}\right.$ and $\left.\mathrm{V}_{\mathrm{b}}=1 \mathrm{~V}\right)$ and cross sectional line profiles of nanowires grown by reactive dewetting of $3 \mathrm{ML}$ iron deposited on a pre-formed $\mathrm{FeO}(111)$ monolayer under $1 \times 10^{-7} \mathrm{mbar}_{2}$ at $\sim 770 \mathrm{~K}$ for 45 minutes; a) shows a maximal field of view image $(0.8 \mu \mathrm{m})$ of two elongated wires of $\sim 4 \mathrm{~nm}$ height and flat upper facets. Some other residual islands co-exist which indicated this is not fully dewet but these also appear with flat upper facets. b) shows an STM image taken at the rectangle in Fig. 6a in which steps are exposed at the edge of the nanowire. A height profile taken along the blue line is shown in the graph with step heights of $0.48 \mathrm{~nm}$; c) shows an STM image taken in the square of Fig. 6a with a line profile across the 
second nanowire. The profile is scaled in height to the $\mathrm{Fe}_{3} \mathrm{O}_{4}(111)$ repeat of $0.48 \mathrm{~nm}$ and shows an exact match for integer multiples of this repeat distance.

In order to probe the structural relationship between the islands formed on the surface and the substrate we adopted modified growth protocols to form fully faceted structures that were small enough to image within the STM. The fully faceted long, narrow and tall nanowires previously discussed become difficult to image by STM as they tend to form double-tipped images very easily which leads to instability (see SI Fig. SI2 for examples). Figure 6a-c show STM images and a line profile across islands and terrace of iron oxide nanostructures dewet from the Re substrate in a single cycle by direct oxidation $\left(5 \times 10^{-7} \mathrm{mbar}, 820 \mathrm{~K}\right)$ of $3 \mathrm{ML}$ of $\mathrm{Fe}$ (see SI for a fig. SI3 2 ML example). Fig. 6a displays the STM topographic image while fig. 6b shows the differential image which highlights step and island edges. The images have been tilted such that the substrate terraces are horizontal and this also coincides with the oxide island upper terraces being flat and parallel to the crystallography of substrate surface. In this region of the substrate there is a high step density and these are readily apparent in the differential image running from top to bottom. The islands do not follow the macroscopic topography, such as predominant step orientation, but are directed by the substrate crystallographic directions. The islands have extended faceted sides along three directions in-plane, one of which coincides with the substrate step structure and is a close packed direction of the substrate, i.e. the $\langle 2 \overline{1} \overline{1} 0\rangle$ family of three equivalent directions, and we therefore conclude the islands align with these directions. In cross section, Fig. 6c, the oxide islands can be seen to form as flat mesas in which the top lies parallel to the terraces of the substrate and is not noticeably influenced by the local step density or orientation, in contrast to some dewetting systems ${ }^{23}$. Fig. $6 \mathrm{~d}$ shows the upper facet of a large 
island (about $11 \mathrm{~nm}$ in height, see SI fig. SI4) grown by multiple cycles of deposition and oxidation $\left(+0.5 \mathrm{ML},+0.5 \mathrm{ML},+1 \mathrm{ML},+1 \mathrm{ML},+1 \mathrm{ML}\right.$ each annealed at $820 \mathrm{~K}$ in $5 \times 10^{-7} \mathrm{mbar}$ $\mathrm{O}_{2}$ ), on a pre-formed $\mathrm{FeO}$ monolayer to give a total of $5 \mathrm{ML}$. The low initial deposition steps ensure that islands nucleate very sparsely and subsequent depositions then grow on those centres leading to fewer but larger islands for the same annealing temperatures and pressures. The islands maintain the very flat upper facet morphology seen for all other islands and nanowires grown, and although they are no longer elongated along the Re surface crystallographic directions the side facets run in the same directions as seen in Figs. 5 and 6a,b. The flatness of the upper terrace can be seen in Fig. 6d, where high resolution STM could be performed on the surface of a large island. This surface is flat but it is also clearly highly structured with small bright clusters decorating a hexagonally reconstructed surface. This morphology is exceptionally similar to the bi-phase surface structure of $\mathrm{Fe}_{3} \mathrm{O}_{4}(111)$ single crystals as seen in Thornton's work ${ }^{24}$ and $\mathrm{Fe}_{3} \mathrm{O}_{4}$ films on $\mathrm{Au}(111)^{25}$. Between the islands the surface is covered by a $\mathrm{FeO}$ wetting layer with a structure very similar to that seen for one monolayer thick films shown in Fig. 2 and is responsible for the XAS signal seen between nanowires in Fig. 4. 

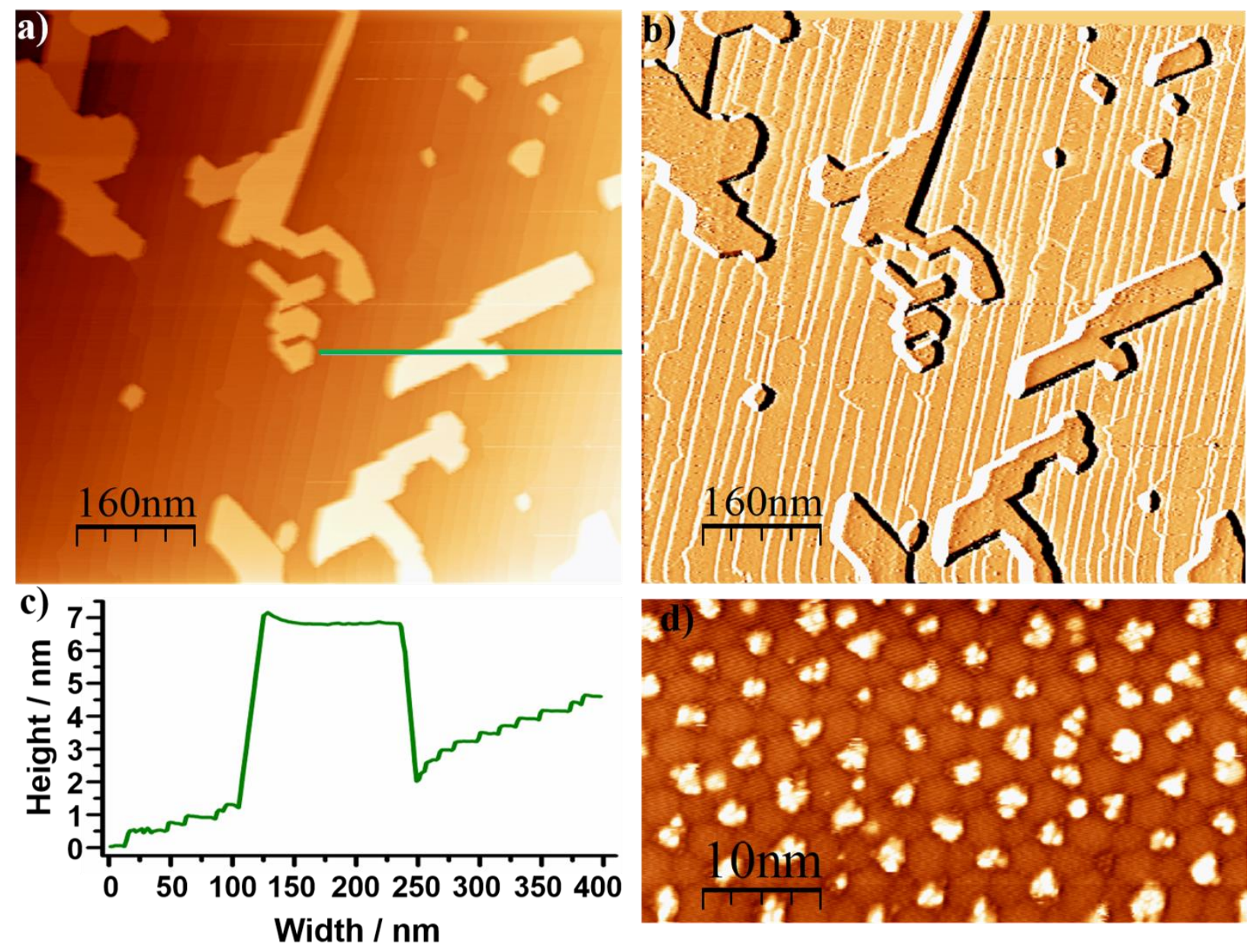

Figure 6. a) STM topographic image $\left(\mathrm{I}_{\mathrm{t}}=0.5 \mathrm{nA}\right.$ and $\left.\mathrm{V}_{\mathrm{b}}=1.5 \mathrm{~V}\right)$ of $\mathrm{FeOx}$ islands dewet from a $\operatorname{Re}(0001)$ surface. b) Differential of a) highlighting terrace steps (and wetting layer grain boundaries) and the flat tops and facetted sides of the crystalline islands. c) Line profile across substrate and island showing atomic steps on the substrate and atomically smooth upper facet of the island parallel to substrate terraces. $d)$ High resolution STM topography $\left(\mathrm{I}_{\mathrm{t}}=0.5 \mathrm{nA}\right.$ and $\mathrm{V}_{\mathrm{b}}=$ $1.5 \mathrm{~V}$ ) from the upper facet of a crystalline nanoisland showing hexagonal array of inclusions as seen in biphase reconstruction of $\mathrm{Fe}_{3} \mathrm{O}_{4}(111)$ single crystals ${ }^{24}$.

The nanowires dewet from the structure have been demonstrated to generate strong dichroism in $\mathrm{XMCD}$ and are therefore ferromagnetic, with the imaging showing that these islands each 
contain a single domain. The STM shows the orientation and phase of the crystalline islands to be related to the substrate crystallography with long axis aligned to close packed substrate directions. The measured step heights of dewet nanowires are consistent with the formation of $\mathrm{Fe}_{3} \mathrm{O}_{4}(111)$ oriented wires with very flat upper terraces. The STM images on top of mesas of large compact islands indicate they are also $\mathrm{Fe}_{3} \mathrm{O}_{4}(111)$ crystallites and all structures form with $\langle 1 \overline{1} 0\rangle$ aligned to the substrate $\langle 2 \overline{1} \overline{1} 0\rangle$. The extended nature of the nanowires, along the $\langle 1 \overline{1} 0\rangle$ direction, with a (111) plane exposed on the surface means that the long side facets are crystallographically inequivalent; one being the (001) plane and the other (110). However, recent DFT calculations of the surface stabilities of magnetite ${ }^{26}$ show that the (110) does not appear in the Wulff construction and that (111) facets would in fact bound this edge. $\{111\}$ surfaces of bulk magnetite are calculated to be slightly less stable than $\{001\}$ and one might imagine that the nanocrystals growing in heavily stepped regions of the substrate with the $\{001\}$ forming the larger facets on the downslope with $\{111\}$ preferred on the upper side facet (and on the mesa surface which is constrained by good epitaxy to the substrate). Currently we cannot directly identify side facet crystallography from our experiments.

One of the primary reasons for dewetting of heteroepitaxial thin films is due to the accumulation of strain energy in the film as it increases in thickness. At a given thickness this strain energy can no longer be accommodated and either structural defects such as dislocations thread through the film or the film breaks up into thicker islands and a thinner wetting layer. In the former case the cost is in the dislocation defect energy and the latter increased surface area and energies plus a potential volumetric strain energy contribution due to thicker islands. In the dewetting to islands some very complex morphologies can arise due to interplays in surface and volume effects ${ }^{27}$. Here we find the dewet oxide islands to be apparently defect free, traversing 
step edges while maintaining a mesa. However, during the dewetting we have also moved from a $\mathrm{Fe} / \mathrm{FeO} / \mathrm{Re}(0001)$ distinctly layered system to one in which the top layer of $\mathrm{Fe}$ is also oxidized. The observed chemical reaction fronts seen here, and in recent published studies, serve to exemplify the complexities of structural and chemical phase transformations ${ }^{14 b, 20}$ in $\mathrm{FeO}_{\mathrm{x}}$ systems and we expect the kinetics of growth to be complex. However, Tersoff and Tromp have developed an approximate expression that describes shape transformations from compact islands into nanowires based upon the energy of dislocation-free strained islands ${ }^{28}$. The model describes the island growth in which energy is minimized by an island narrowing in width while it extends in length along an orthogonal direction. During this process the island height needs to remain constrained for the model to operate successfully. The model has been shown to describe a variety of systems including $\mathrm{Ag}$ nanowires on $\mathrm{Si}(001)$, elongated anatase $\mathrm{TiO}_{2}(001)$ islands on $\mathrm{SrTiO}_{3}(001){ }^{27 b}$ and elongated clusters of $\mathrm{CoSi}_{2}$ on $\mathrm{Si}(001){ }^{29}$. It is probable that a similar mechanism is responsible for the observations in our studies, in which compact oxide islands elongate and narrow to form extended wires. The height constraint is due to a relatively slow diffusion path to the top of the nanowires compared with a rapid diffusion path to the base. Alternatively one could argue that the growth facet at the end of the $\mathrm{Fe}_{3} \mathrm{O}_{4}$ nanowire is particularly reactive and hence the growth shape is dominated by the kinetics of facet attachment energies rather than equilibrium thermodynamics. However, such a model could be expected to lead to growth directed by the substrate step density, as is seen in Fe growth on $\mathrm{Mo}(110)^{30}$. We note that at the oxygen chemical potentials employed here that magnetite surfaces are predicted by DFT to oxidise significantly and it is also not clear whether the bulk of the nanowire could truly be described as magnetite due to their very small size in cross-section ${ }^{26}$. It is possible that this shape transformation from compact islands to high aspect ratio wires is accompanied by a 
change in oxide stoichiometry, possibly forming maghemite which has the magnetite structure but with $\mathrm{Fe}^{2+}$ vacancies. Clearly it would be of interest to search the P-T parameter space experimentally to identify critical island sizes that could transform to functional magnetic nanowires and to be able to simultaneously structurally identify the phase of these very thin wires. Having experimentally identified a new morphology in the heteroepitaxial $\mathrm{FeO}_{\mathrm{x}}$ system here it is hoped that further theoretical modelling of surface, interface and bulk energies may reach sufficient fidelity to enable the prediction of critical island sizes and give rise to better understanding and control of surface supported nanomaterial growth.

In conclusion, we have shown that high aspect ratio iron oxide nanowires of several microns length and less than $40 \mathrm{~nm}$ width can be grown by the reactive dewetting of an iron rich oxide film supported on a rhenium surface. The nanowires are formed in registry with the substrate appearing to be $\pm 5^{\circ}$ from a substrate high symmetry direction in large area LEEM images while in STM over short distances on smaller islands these appear to be in epitaxy with the close packed rhenium. The nanowires show distinctive XMCD contrast as a result of each being composed of a single ferro (or ferri) magnetic domain and hence are of potential use in magnetic device and spintronic applications. Step edges at the periphery of the nanowires are consistent with a magnetite structure oriented with the (111) surface facet uppermost. As the $\mathrm{FeO}$ wetting layer that accompanies these nanostructures is commonly seen on a variety of other hexagonal substrates it is thought that the growth phenomena is also likely to occur on other substrates with similar lattice constants ( $\mathrm{Pt}, \mathrm{Pd}$ etc). It is further probable that the dewetting follows the Tersoff and Tromp model of shape transformation, from compact islands into nanowires, based upon the energy of dislocation-free strained islands.

\section{Corresponding Author}


*r.a.bennett@reading.ac.uk

\section{Author Contributions}

The manuscript was written through contributions of all authors. All authors have given approval to the final version of the manuscript.

\section{ACKNOWLEDGMENT}

We would like to thank the STFC for synchrotron beamtime and colleagues at the Diamond synchrotron for supporting the work and also the Royal Society / Wolfson Foundation for facilities refurbishment in Reading. HAE would like to thank the EPSRC for studentship funding. We acknowledge the expertise of Dr Peter Harris of the EMLab at the University of Reading who acquired the SEM images of our nanostructured surface.

Insert Table of Contents Graphic and Synopsis Here
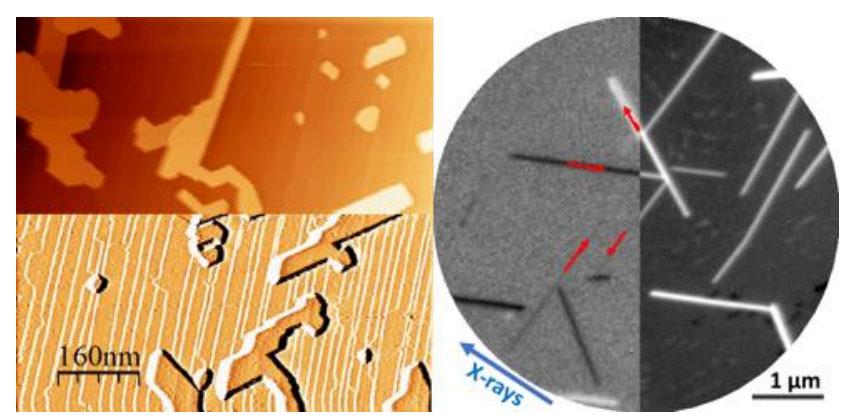

Supporting Information 
We provide an expanded experimental description of the methods used and the analysis of XMCD imaging. We also show movies recorded during 1) the growth of Fe on the clean $\operatorname{Re}(0001)$ surface; 2$)$ the formation of nanowires after a reaction front has passed across the $\mathrm{Fe}$ on $\mathrm{FeO}_{\mathrm{x}}$ surface; 3 ) the formation of nanowires without a surface reaction front traversing the crystal; 4) the LEED-IV patterns visible prior to nanowire formation and 5) the images accompanying the XAS measurements of the nanowires. We also include four sets of images that provide supporting information about the island growth.

Supporting Information Available: This material is available free of charge via the Internet at http://pubs.acs.org.1. Zhang, Z.; Satpathy, S., Electron states, magnetism, and the Verwey transition in magnetite. Phys. Rev. B 1991, 44 (24), 13319-13331.

2. $\quad$ Parkinson, G. S., Iron oxide surfaces. Surface Science Reports 2016, 71 (1), 272-365.

3. Senn, M. S.; Wright, J. P.; Attfield, J. P., Charge order and three-site distortions in the Verwey structure of magnetite. Nature 2012, 481 (7380), 173-176.

4. Rezníček, R.; Chlan, V.; Štěpánková, H.; Novák, P., Hyperfine field and electronic structure of magnetite below the Verwey transition. Phys. Rev. B 2015, 91 (12), 125134. 5. $\quad$ Lee, J.; Kwon, S. G.; Park, J.-G.; Hyeon, T., Size Dependence of Metal-Insulator Transition in Stoichiometric $\mathrm{Fe}_{3} \mathrm{O}_{4}$ Nanocrystals. Nano Letters 2015, 15 (7), 4337-4342. 6. $\quad$ Parkinson, G. S.; Diebold, U.; Tang, J.; Malkinski, L., Tailoring the Interface Properties of Magnetite for Spintronics, Advanced Magnetic Materials, Dr. Leszek Malkinski (Ed.). InTech: 2012.

7. $\quad$ Liao, Z.-M.; Li, Y.-D.; Xu, J.; Zhang, J.-M.; Xia, K.; Yu, D.-P., Spin-Filter Effect in Magnetite Nanowire. Nano Letters 2006, 6 (6), 1087-1091.

8. Yun, J.-G.; Lee, Y.-M.; Lee, W.-J.; Kim, C.-S.; Yoon, S.-G., Selective growth of pure magnetite thin films and/or nanowires grown in situ at a low temperature by pulsed laser deposition. Journal of Materials Chemistry C 2013, 1 (10), 1977-1982.

9. Takahashi, R.; Misumi, H.; Yamamoto, T.; Lippmaa, M., Spontaneous Growth of StrainFree Magnetite Nanocrystals via Temperature-Driven Dewetting. Crystal Growth \& Design 2014, 14 (3), 1264-1271.

10. Tauster, S. J.; Fung, S. C.; Garten, R. L., Strong metal-support interactions. Group 8 noble metals supported on titanium dioxide. Journal of the American Chemical Society 1978, $100(1), 170-175$.

11. Braunschweig, E. J.; Logan, A. D.; Datye, A. K.; Smith, D. J., Reversibility of strong metal-support interactions on RhTiO2. Journal of Catalysis 1989, 118 (1), 227-237.

12. Datye, A. K.; Kalakkad, D. S.; Yao, M. H.; Smith, D. J., Comparison of Metal-Support Interactions in $\mathrm{Pt} / \mathrm{TiO} 2$ and $\mathrm{Pt} / \mathrm{CeO} 2$. Journal of Catalysis 1995, 155 (1), 148-153.

13. (a) Willinger, M. G.; Zhang, W.; Bondarchuk, O.; Shaikhutdinov, S.; Freund, H.-J.; Schlögl, R., A Case of Strong Metal-Support Interactions: Combining Advanced Microscopy and Model Systems to Elucidate the Atomic Structure of Interfaces. Angewandte Chemie International Edition 2014, 53 (23), 5998-6001; (b) Majzik, Z.; Balázs, N.; Berkó, A., Ordered 
SMSI Decoration Layer on $\mathrm{Rh}$ Nanoparticles Grown on $\mathrm{TiO}_{2}(110)$ Surface. The Journal of Physical Chemistry C 2011, 115 (19), 9535-9544; (c) Bennett, R. A.; Pang, C. L.; Perkins, N.; Smith, R. D.; Morrall, P.; Kvon, R. I.; Bowker, M., Surface Structures in the SMSI State; Pd on $(1 \times 2)$ Reconstructed $\mathrm{TiO}_{2}(110)$. The Journal of Physical Chemistry B 2002, 106 (18), 46884696; (d) Silly, F.; Castell, M. R., Encapsulated Pd Nanocrystals Supported by NanolineStructured $\mathrm{SrTiO}_{3}(001)$. The Journal of Physical Chemistry B 2005, 109 (25), 12316-12319. 14. (a) Qiu, H.; Staemmler, V.; Kuhlenbeck, H.; Bauer, E.; Freund, H.-J., Weak thermal reduction of biphase $\mathrm{Fe}_{2} \mathrm{O}_{3}(0001)$ films grown on $\mathrm{Pt}(111)$ : Sub-surface $\mathrm{Fe}^{2+}$ formation. Surface Science 2015, 641, 30-36; (b) Genuzio, F.; Sala, A.; Schmidt, T.; Menzel, D.; Freund, H.-J., Interconversion of $\alpha-\mathrm{Fe}_{2} \mathrm{O}_{3}$ and $\mathrm{Fe}_{3} \mathrm{O}_{4}$ Thin Films: Mechanisms, Morphology, and Evidence for Unexpected Substrate Participation. The Journal of Physical Chemistry C 2014, 118 (50), 2906829076; (c) Ketteler, G.; Ranke, W., Self-assembled periodic $\mathrm{Fe}_{3} \mathrm{O}_{4}$ nanostructures in ultrathin FeO(111) films on Ru(0001). Phys. Rev. B 2002, 66 (3), 033405; (d) Santos, B.; Loginova, E.; Mascaraque, A.; Schmid, A. K.; McCarty, K. F.; Figuera, J. d. 1., Structure and magnetism in ultrathin iron oxides characterized by low energy electron microscopy. Journal of Physics: Condensed Matter 2009, 21 (31), 314011.

15. Etman, H. A.; Zheleva, Z. V.; Held, G.; Bennett, R. A., Epitaxial Growth of Ultrathin Palladium Films on $\operatorname{Re}\{0001\}$. The Journal of Physical Chemistry C 2011, 115 (10), 4191-4199. 16. Horcas, I.; Fernández, R.; Gómez-Rodríguez, J. M.; Colchero, J.; Gómez-Herrero, J.; Baro, A. M., WSXM: A software for scanning probe microscopy and a tool for nanotechnology. Review of Scientific Instruments 2007, 78 (1), 013705.

17. Ritter, M.; Ranke, W.; Weiss, W., Growth and structure of ultrathin FeO films on Pt(111) studied by STM and LEED. Phys. Rev. B 1998, 57 (12), 7240-7251.

18. Merte, L. R.; Grabow, L. C.; Peng, G.; Knudsen, J.; Zeuthen, H.; Kudernatsch, W.; Porsgaard, S.; Lægsgaard, E.; Mavrikakis, M.; Besenbacher, F., Tip-Dependent Scanning Tunneling Microscopy Imaging of Ultrathin FeO Films on Pt(111). The Journal of Physical Chemistry C 2011, 115 (5), 2089-2099.

19. Berdunov, N.; Mariotto, G.; Murphy, S.; Balakrishnan, K.; Shvets, I. V., Self-assembly of iron nanoclusters on the $\mathrm{Fe}_{3} \mathrm{O}_{4}(111)$ superstructured surface. Phys. Rev. B 2005, 71 (11), 113406. 20. Genuzio, F.; Sala, A.; Schmidt, T.; Menzel, D.; Freund, H. J., Phase transformations in thin iron oxide films: Spectromicroscopic study of velocity and shape of the reaction fronts. Surface Science 2016, 648, 177-187.

21. Monti, M.; Santos, B.; Mascaraque, A.; Rodríguez de la Fuente, O.; Niño, M. A.; Menteş, T. O.; Locatelli, A.; McCarty, K. F.; Marco, J. F.; de la Figuera, J., Oxidation Pathways in Bicomponent Ultrathin Iron Oxide Films. The Journal of Physical Chemistry C 2012, 116 (21), 11539-11547.

22. Regan, T. J.; Ohldag, H.; Stamm, C.; Nolting, F.; Luning, J.; Stohr, J.; White, R. L., Chemical effects at metal/oxide interfaces studied by x-ray-absorption spectroscopy. Phys. Rev. B 2001, 64 (21), 214422.

23. Ling, W. L.; Giessel, T.; Thürmer, K.; Hwang, R. Q.; Bartelt, N. C.; McCarty, K. F., Crucial role of substrate steps in de-wetting of crystalline thin films. Surface Science 2004, 570 (3), L297-L303.

24. Condon, N. G.; Leibsle, F. M.; Parker, T.; Lennie, A. R.; Vaughan, D. J.; Thornton, G., Biphase ordering on $\mathrm{Fe}_{3} \mathrm{O}_{4}(111)$. Phys. Rev. B 1997, 55 (23), 15885-15894.

25. Deng, X.; Lee, J.; Matranga, C., Preparation and characterization of $\mathrm{Fe}_{3} \mathrm{O}_{4}(111)$ nanoparticles and thin films on Au(111). Surface Science 2010, 604 (7), 627-632. 
26. Santos-Carballal, D.; Roldan, A.; Grau-Crespo, R.; de Leeuw, N. H., A DFT study of the structures, stabilities and redox behaviour of the major surfaces of magnetite $\mathrm{Fe}_{3} \mathrm{O}_{4}$. Physical Chemistry Chemical Physics 2014, 16 (39), 21082-21097.

27. (a) Bennett, R. A.; Mulley, J. S.; Etman, H. A.; Sparkes, A.; Eralp, T.; Held, G.; Cavill, S. A.; Dhesi, S. S., Chromium nanostructures formed by dewetting of heteroepitaxial films on W(100). Phys. Rev. B 2012, 86 (4), 045454; (b) Marshall, M. S. J.; Castell, M. R., Shape Transitions of Epitaxial Islands during Strained Layer Growth: Anatase $\mathrm{TiO}_{2}(001)$ on $\mathrm{SrTiO}_{3}(001)$. Phys. Rev. Lett. 2009, 102 (14), 146102.

28. Tersoff, J.; Tromp, R. M., Shape transition in growth of strained islands: Spontaneous formation of quantum wires. Phys. Rev. Lett. 1993, 70 (18), 2782-2785.

29. Brongersma, S. H.; Castell, M. R.; Perovic, D. D.; Zinke-Allmang, M., Stress-Induced Shape Transition of $\mathrm{CoSi}_{2}$ Clusters on Si(100). Phys. Rev. Lett. 1998, 80 (17), 3795-3798.

30. Shvets, I. V.; Murphy, S.; Kalinin, V., Nanowedge island formation on Mo(110). Surface Science 2007, 601 (15), 3169-3178. 